\title{
Second-Order Logic and Foundations of Mathematics*
}

\author{
Jouko Väänänen ${ }^{\dagger}$ \\ Department of Mathematics \\ University of Helsinki \\ Helsinki, Finland
}

August 21, 2001

\begin{abstract}
We discuss the differences between first-order set theory and secondorder logic as a foundation for mathematics. We analyse these languages in terms of two levels of formalization. The analysis shows that if second-order logic is understood in its full semantics capable of characterizing categorically central mathematical concepts, it relies entirely on informal reasoning. On the other hand, if it is given a weak semantics, it loses its power in expressing concepts categorically. First-order set theory and second-order logic are not radically different: the latter is a major fragment of the former.
\end{abstract}

\section{Introduction}

Second-order logic differs from the usual first-order predicate calculus in that it has variables and quantifiers not only for individuals but also for subsets of the universe (sometimes variables for $n$-ary relations as well, but this is not important in this context). The deductive calculus $\mathrm{DED}_{2}$ of secondorder logic is based on rules and axioms ([Hen50]) which guarantee that the

*I am grateful to Juliette Kennedy for many helpful discussions while developing the ideas of this paper.

${ }^{\dagger}$ Research partially supported by grant 40734 of the Academy of Finland. 
quantifiers range at least over definable subsets. As to the semantics, there are two versions of models: Suppose $\mathfrak{A}$ is an ordinary first-order structure and $\mathcal{S}$ is a set of subsets of the domain $A$ of $\mathfrak{A}$. The idea is that the set-variables range over $\mathcal{S}$ :

$$
\langle\mathfrak{A}, \mathcal{S}\rangle \models \exists X \phi(X) \Longleftrightarrow(\exists S \in \mathcal{S})(\langle\mathfrak{A}, \mathcal{S}\rangle \models \phi(S)) .
$$

We call $\langle\mathfrak{A}, \mathcal{S}\rangle$ a Henkin model, if $\langle\mathfrak{A}, \mathcal{S}\rangle$ satisfies the axioms of $\mathrm{DED}_{2}$ and truth in $\langle\mathfrak{A}, \mathcal{S}\rangle$ is preserved by the rules of $\mathrm{DED}_{2}$. We call this semantics of second-order logic the Henkin semantics and second-order logic with the Henkin semantics the Henkin second-order logic. There is a special class of Henkin models, namely those $\langle\mathfrak{A}, \mathcal{S}\rangle$ where $\mathcal{S}$ is the set of all subsets of $A$. We call these full models. We call this semantics of second-order logic the full semantics and second-order logic with the full semantics the full second-order logic. ${ }^{1}$.

The following facts are the main features of second-order logic:

- The Completeness Theorem: A sentence is provable in $\mathrm{DED}_{2}$ if and only if it holds in all Henkin models ([Hen50]).

- The Löwenheim-Skolem Theorem: A sentence with an infinite Henkin model has a countable Henkin model.

- The Compactness Theorem: A set of sentences, every finite subset of which has a Henkin model, has itself a Henkin model.

- The Incompleteness Theorem: Neither $\mathrm{DED}_{2}$ nor any other effectively given deductive calculus is complete for full models, that is, there are always sentences which are true in all full models but which are unprovable.

- Failure of the Compactness Theorem for full models.

- Failure of the Löwenheim-Skolem Theorem for full models.

- There is a finite second-order axiom system $\mathrm{Z}_{2}$ such that the semiring $\mathbb{N}$ of natural numbers is the only full model (up to isomorphism) of $Z_{2}$.

\footnotetext{
${ }^{1}$ Also other kinds of models have been studied. In weak second-order logic (see e.g. [Mos61]) one considers only models $\langle\mathfrak{A}, \mathcal{S}\rangle$ where $\mathcal{S}$ is the set of all finite subsets of $A$. One can also limit $\mathcal{S}$ to the set of all countable subsets of $A$. Finally, one may limit $\mathcal{S}$ to the set of all first-order definable subsets of $A([\operatorname{Lin} 73])$.
} 
- There is a finite second-order axiom system $\mathrm{RCF}_{2}$ such that the field $\mathbb{R}$ of real numbers is the only (up to isomorphism) full model of $\mathrm{RCF}_{2}$.

As the above facts demonstrate, it is highly critical what kind of semantics one uses for second-order logic. One semantics gives beautiful categoricity $^{2}$ results. Another semantics gives beautiful model-theoretic methods. Mathematical logic has results such as Gödel's Incompleteness Theorem that analyse the reasons why there seems to be a dichotomy: either categoricity or axiomatization. I will argue in this paper that if second-order logic is used in formalizing or axiomatizing mathematics, the choice of semantics is irrelevant: it cannot meaningfully be asked whether one should use Henkin semantics or full semantics. This question arises only if we formalize second-order logic after we have formalized basic mathematical concepts needed for semantics. A choice between the Henkin second-order logic and the full second-order logic as a primary formalization of mathematics cannot be made; they both come out the same.

If one wants to use the full second-order logic for formalizing mathematical proofs, the best formalization of it so far is the Henkin second-order logic. In other words, I claim, that if two people started using second-order logic for formalizing mathematical proofs, person $F$ with the full second-order logic and person $H$ with the Henkin second-order logic, we would not be able to see any difference. There would be the secondary difference, that if mathematicians were unable to decide whether some statement (like the Continuum Hypothesis) is true or false, person $H$ could formalize the deductive calculus $\mathrm{DED}_{2}$, and show that the statement is independent of $\mathrm{DED}_{2}$. This would be a clear message that something new is needed for deciding the statement. Person $F$ is a priori not in such a position and would be unable to tell why the statement eludes proof or disproof.

The situation is similar to that regarding the cumulative hierarchy and Zermelo-Fraenkel set theory. We can informally define the sets

$$
\begin{aligned}
& R_{0}=\emptyset \\
& R_{\alpha+1}=\text { the set of all subsets of } R_{\alpha} \\
& R_{\nu}=\bigcup_{\alpha<\nu} R_{\alpha} \text { for } \nu \text { limit, }
\end{aligned}
$$

and regard their union $V$ as the standard model of set theory. We can do the same more formally inside ZFC set theory and prove in ZFC that indeed every

\footnotetext{
${ }^{2}$ An axiom system is said to be categorical if it has only one model up to isomorphism.
} 
set is in (what ZFC thinks is ) $V$. We cannot meaningfully ask whether the $V$ as defined in ZFC is the real $V$. But if we reformalize ZFC inside ZFC, then we can note that the reformalized ZFC, call it $\mathbf{Z F C}$, has countable models and hence cannot be categorical. We have the following match:

$$
\frac{\text { ZFC }}{V}=\frac{\text { Henkin models }}{\text { Full models }} \text {. }
$$

As with second-order logic, we cannot really choose whether we axiomatize mathematics using $V$ or ZFC. The result is the same in both cases, as ZFC is the best attempt so far to use $V$ as an axiomatization of mathematics. If we think of the truth or falsity of the Continuum Hypothesis $(\mathrm{CH})$ in $V$, Cohen's proof of the independence of $\mathrm{CH}$ from ZFC, with all the information about models of set theory that came with the proof, is a huge step toward understanding why $\mathrm{CH}$ has not yet been settled in $V$. Likewise, the study of Henkin models of second-order artihmetic (see e.g. [Sim99]) isolate reasons why some results of number theory or analysis are hard to prove.

\section{Preliminary example}

Mathematicians argue exactly but informally. This has worked well for centuries. However, if we want to understand the way mathematicians argue, it is necessary to formalize basic concepts such as the concepts of language and criteria of truth.

We study two metatheories of mathematics: first-order set theory and second-order logic. It is often said (e.g. [Sha91]), that second-order logic is better than first-order set theory because it can in its full semantics axiomatize categorically $\mathbb{N}$ and $\mathbb{R}$, while first-order axiomatization of set theory admits non-standard, e.g. countable models. We show below that this difference is illusory. If second-order logic is construed as our primitive logic, one cannot say whether it has full semantics or Henkin semantics, nor can we meaningfully say whether it axiomatizes categorically $\mathbb{N}$ and $\mathbb{R}$. So there is no difference between the two logics: first-order set theory is merely the result of extending second-order logic to transfinitely high types. Such an extension is known to be conservative ${ }^{3}[$ Hin55, Mon65a].

\footnotetext{
${ }^{3}$ More exactly, higher order logic can be reduced to second-order logic. To decide how high order logic can be reduced depends only on how long well-ordered order-types are definable in second-order logic.
} 
In both first-order set theory and second-order logic we think that there is a universe (or many universes) of mathematical objects where the sentences of our logic have a meaning. This meaning cannot be defined mathematically. Any attempt to do so ends up in a vicious circle. We cannot even ask meaningfully whether the universe is unique or a multitude of many universes. We simply do not have a language for talking about the universe or the universes as objects that could be identical to each other.

As a concrete example of a mathematical proof and its formalization, let us consider the following:

Bolzano's Theorem: Every continuous function on [0,1], which has a negative value at 0 and a positive value at 1 , has the value 0 at some point on $(0,1)$.

Proof: Let the function be $f$. Consider the set

$$
X=\{x \in(0,1): f(x)<0\} .
$$

This set is bounded from above by 1 and hence has a supremum. Call the supremum $a$. If $f(a)<0$, then by continuity there is a neighborhood of $a$ on $(0,1)$ in which $f(x)<0$. Hence $a$ could not be the supremum of $X$. If $f(a)>0$, then there is a neighborhood in which $f(x)>0$, so again $X$ could not be the supremum. Hence $f(a)=0$.

A formalization of this in first-order set theory is based on the Completeness Property

$$
\begin{aligned}
& \forall u \subseteq \mathbb{R}((u \neq \emptyset \wedge \exists z \forall x(x \in u \rightarrow x \leq z)) \\
& \rightarrow \exists z(\forall x(x \in u \rightarrow x \leq z) \wedge \\
& \left.\forall z^{\prime}\left(\forall x\left(x \in u \rightarrow x \leq z^{\prime}\right)\right) \rightarrow z \leq z^{\prime}\right)
\end{aligned}
$$

of the set-theoretic construction of the reals (e.g. as Cauchy-sequences or Dedekind cuts) and on an instance of the Separation Schema

$$
\exists z \forall x(x \in z \leftrightarrow(\phi(x) \wedge x \in \mathbb{R})),
$$

where $\phi(x)$ is chosen to be the formula $f(x)<0 \wedge 0<x<1$. 
A formalization of the same proof in second-order logic would use the following Continuity Axiom of the axiomatization of the structure of reals in second-order logic:

$$
\begin{aligned}
& \forall X((\exists x X(x) \wedge \exists z \forall x(X(x) \rightarrow x \leq z)) \\
& \rightarrow \exists z(\forall x(X(x) \rightarrow x \leq z) \wedge \\
& \left.\forall z^{\prime}\left(\forall x\left(X(x) \rightarrow x \leq z^{\prime}\right)\right) \rightarrow z \leq z^{\prime}\right)
\end{aligned}
$$

We instantiate the second-order universal quantifier $\forall X$ by substituting the set $\{x \in(0,1): f(x)<0\}$ into $X$. We do not need the full semantics as the set $\{x \in(0,1): f(x)<0\}$ is definable by $\phi(x)$. Thus we can prove Bolzano's Theorem in Henkin's complete axiom system ([Hen50]) for second-order logic.

There is not much difference between the two formalizations of the informal proof of Bolzano's Theorem. The second-order logic proof is like a pared down version of the more lavish set-theoretic proof. All we need is axioms about definable sets of reals, so there is no need to postulate all of set theory.

\section{Informal reasoning, formalization}

Informal mathematical reasoning, appealing to intuition, is the only process, if any such exists, which puts us in contact with mathematical truth. In the late 19th century mathematics had reached such elaboration that mere intuition was not a sufficient guiding principle any more. Properties of point-sets and continuous functions such as space-filling curves, nowhere differentiable continuous functions, paradoxical decompositions of the sphere and so forth, seemed to defy intuition. It was suggested that the human mind may not be adequate to dealing with the subtleties of infinite sets and mathematical reasoning should therefore be limited to the area of finite sets and finite operations. Since infinite sets however abound in mathematics, everything beyond finite was to be formalized and axiomatized into a game on finite strings of symbols.

One of the developments that exposed conceptual confusions in the early 20 th century was the appearance of paradoxes ${ }^{4}$. These examples center

\footnotetext{
${ }^{4}$ Richard's paradox: "The smallest natural number not definable by nine words is definable by nine words." Russell's paradox: "The set of sets which are not elements of themselves is not an element of itself, hence is an element of itself" Burali Forti paradox: "The set of all well-ordered sets can be well-ordered under order-preserving
} 
around the concepts of definability and large sets, and fail to touch upon topics in classical mathematics, such as analysis, algebra and number theory. For the foundations of mathematics and attempts to formalize mathematics they presented a challenge. Formalization does not per se remove paradoxes, rather the contrary. It is quite possible that a formalization of a part of mathematics involves a formalization of a paradox. However, formal methods are now known for the avoidance of particular paradoxes. To avoid Richard's paradox one limits the induction axioms to sets definable in a certain formal language. The paradox then turns into a proof that the set of definable numbers is not itself definable. For the other paradoxes above, one distinguishes between a set and a class, and the paradoxes then simply demonstrate that certain classes are not sets.

Another reason for formalization is the need to understand basic principles, which, though probably consistent, seem to elude clarity. The axiom of choice is a good example. Projective determinacy is a more recent example. At the lower end of complexity we have $\Pi_{2}^{1}$-comprehension. I will argue below that first-order logic serves us better in this kind of formalization than second-order logic.

A general reason for formalization, related to understanding basic principles, is the need to develop meaning theory for mathematics. The idea that mathematics has no meaning at all contradicts the basic experience of doing mathematics. It can be argued that the experience of meaning is an illusion, which cannot be subjected to exact study. If this were the case, it is conceivable that an attempt to study the meaning would manifest this, ending in impossibility or incredibility.

But alas, we cannot formalize the real content of informal reasoning. This is a fundamental fact. Wittgenstein ends his Tractatus with the words

"My propositions serve as elucidations in the following way: anyone who understands me eventually recognizes them as nonsensical, when he has used them-as steps-to climb up beyond them."

Formalization always involves a leap of faith - "ladders" - to cross a gaping crevice between what is intended and what is achieved. Tarski and Gödel proved exact results to the same effect. In particular we cannot hope to find conclusive security against paradoxes via formalization.

embeddings. Hence it is isomorphic to an initial segment of itself and therefore not wellordered" Cantor's paradox : "The powerset $P$ of the set $A$ of all sets has cardinality greater than $A$, although $P$ is a subset of $A . "$ 
A lot of effort is put into reducing consistency statements of formal systems to a more transparent form, e.g. transfinite induction, where intuition could be more reliable. This will always leave open the question whether a lack of balance in this reduction is a sign of lack of consistency or a sign of inherent limitation of intuition.

Informal reasoning will remain the guiding line in mathematics; formal methods involve always a speculative element to be convincing, as we cannot fix the meaning of our formal expressions. This is paradoxical. Surely informal reasoning about infinite objects feels more like speculation than formal reasoning, where everything is spelled out in axioms. But in the latter case we can only speculate whether our formalization captures what we intend. I argue below that this is true whether we use first-order or second-order formalization.

\section{Urlogic}

Formalization of mathematics involves defining a formal language with some intended meaning. Let us call this language urlogic ${ }^{5}$. The idea is that urlogic is the most primitive formal language we use to study the process of doing mathematics.

Urlogic is the aspect of mathematicians' activity that consists of just writing down finite strings of symbols - sentences - according to some fixed rules. Those sentences are sentences of urlogic. Whether a string of symbols is a sentence of urlogic should be totally unproblematic.

Intuitively these sentences have a meaning in the universe (or universes) of mathematical objects. Some sentences are said to be true by virtue of expressing a true proposition in this universe. The whole point of mathematics is the truth of its sentences, not their form. It is also the truth which is problematic and calls for formalization. We discuss formalization of truth in Section 5.

If one asks a mathematician why he or she calls a sentence $\phi$ on the blackboard "true" the answer is not that $\phi$ is true because its meaning is a true proposition of the universe of mathematical objects. The answer would be that $\phi$ is true because it can be proved from first principles. These first

\footnotetext{
${ }^{5}$ The word "metatheory" is often used for a similar notion. When we introduce the concept of "object language" in Section 5, we could call urlogic a metatheory of the object language.
} 
principles are called "first" because they are considered self-evident (and their further elucidation is left to logicians). Examples of such first principles are (1) and (2) above. Further examples are the Archimedian Axiom for the reals $^{6}$, and the Induction Axiom for natural numbers ${ }^{7}$.

How difficult should it be to tell if some principle is a first principle or not? In order to really be on the most primitive level, it should be a nonmathematical question whether a statement has actually been listed as a first principle or not. That we may disagree as to what should be listed there or not is another matter. Thus we call some statements of urlogic axioms and regard them as first principles that are accepted true by agreement. No mathematics should be involved in checking that a string is indeed in the list of axioms, it is merely a question of comparing finite strings of symbols to a list of such strings.

When a mathematician is asked to tell why a sentence is true, he or she appeals to what follows from the first principles, the axioms. What does he or she mean by "follow"? At its simplest, this means appealing to such basic rules of logic as

$$
\text { From } \phi \text { and } \phi \rightarrow \psi \text { follows } \psi \text {. }
$$

$$
\text { From } \forall x \phi(x) \text { follows } \phi(t) \text { for each term } t \text {. }
$$

As the example of the proof of Bolzano's Theorem showed, it is important to discuss what is meant by "term" here. In urlogic we are dealing with finite strings of symbols. If we want to derive the string $\phi(t)$ from the string $\forall x \phi(x)$ by substitution of $t$ into $x, t$ must be a string as well. We cannot in this context substitute a set $t$ into $x$. That would not make sense, because the result would not be a finite string. Thus $t$ has to be a finite string itself, a variable, a constant or a complex term arising from function symbols or other operations such as Hilbert's $\epsilon$-operation or Church's $\lambda$-notation.

There may be other rules of derivation than (3) and (4), for example the $\omega$-rule:

$$
\text { From } \phi(\underline{n}) \text { for all } n=0,1,2, \ldots \text { follows } \forall x(N(x) \rightarrow \phi(x)) \text {, }
$$

where $\underline{n}$ is a term denoting the natural number $n$ and $N$ is the name for the set of natural numbers. The problem with this rule as a rule of urlogic

\footnotetext{
${ }^{6}$ Every real number is less than 1 or $1+1$ or $1+1+1$ or $\ldots$.

${ }^{7}$ Every set of natural numbers, which contains 0 , and which contains the successor of each of its elements, contains all natural numbers.
} 
is that derivations become infinite. When the $\omega$-rule is used repeatedly, a whole infinite tree of sentences emerges. We cannot say that this is the most primitive level of analyzing the process of doing mathematics. The concept of derivation as an infinite tree calls for an analysis too. It is only when derivations are finite that we can say that we are at the root of things, where we cannot conceive of a more primitive concept of derivation ${ }^{8}$.

In summary, urlogic has the following characteristics:

- Sentences of urlogic are finite strings of symbols. That a string of symbols is a sentence of urlogic, is a non-mathematical judgement.

- Some sentences are accepted as axioms. That a sentence is an axiom, is a non-mathematical judgement.

- Derivations are made from axioms. The derivations obey certain rules of proof. That a derivation obeys the rules of proof, is a non-mathematical judgement.

- Derived sentences can be asserted as facts.

In classical mathematics the law of excluded middle

$$
\phi \vee \neg \phi
$$

is asserted as a fact, but it is not the case, and it does not follow from this, that given any sentence $\phi$, either $\phi$ or $\neg \phi$ can be asserted as a fact. It may be that neither has been derived yet, and it may be that neither can be derived because of some weakness in the axioms. By Gödel's Incompleteness Theorem it seems unavoidable that there always are such sentences $\phi$.

If we take first-order set theory as the urlogic, the sentences of urlogic are sentences of first-order predicate logic with identity, with the binary predicate symbol $\in$ as the only non-logical symbol. The axioms are the usual rules of first-order logic augmented with the Zermelo-Fraenkel axioms ZFC of set theory. On the informal level we interpret the sentences of this urlogic as propositions about mathematical objects construed as sets. As mathematical objects are not a priori sets, a certain reinterpretation is called for in our mind. This however is in harmony with the general ideology in mathematics

\footnotetext{
${ }^{8}$ In fact, a more primitive notion would be a "surveyable" proof, a proof we can understand as opposed to a random sequence of sentences which happens to obey the rules of proof.
} 
that only the mutual relationships of mathematical objects really matter, not what the objects are made of.

In the case of second-order logic the sentences of urlogic are the sentences of second-order predicate logic. Depending on the context, the non-logical vocabulary may consist of symbols for the arithmetic of natural numbers, arithmetic of real numbers, and so forth. Montague [Mon65b] gives secondorder Peano axioms $\mathrm{Z}_{2}$ for number theory, and second-order axioms $\mathrm{RCF}_{2}$ for real closed fields. For full second-order logic there is a notion of "semantical" derivation:

We can derive $\psi$ from $\phi$ if every model of $\phi$ is a model of $\psi$.

However, we cannot accept this rule in urlogic, since scanning through all models of $\phi$ is a highly mathematical act. So we have to settle with the rules $\mathrm{DED}_{2}$, such as those presented in [Hen50], most notably

$$
\text { From } \forall X \phi(X) \text { follows } \phi\left(\left\{x: \psi\left(x, y_{1}, \ldots y_{n}\right)\right\}\right)
$$

for each formula $\psi\left(x, y_{1}, \ldots, y_{n}\right)$, plus perhaps some new ones that we do not know yet. There are technical results, e.g. in [Hen50], to the effect that second-order logic with rules like (7) admit non-standard models where the second-order variables do not range over all subsets of the domain. These are not relevant here, however, as we have not formalized the semantics of second-order logic. So on the level of urlogic it does not make sense to ask if the models of our sentences are standard or non-standard. The only "model" we have is the world of mathematical objects, and we have no langauge for making a statement to the effect that the universe is non-standard. It is more natural to think that it is standard. So in this sense second-order logic as the urlogic has the full semantics. On the other hand, the rules of proof of second-order logic as the urlogic give it the appearance of Henkin second-order logic or first-order set theory. The truth is, if we only consider urlogic, we cannot tell first-order logic and second-order logic, weak and full semantics, really apart. We cannot subject urlogic to mathematical scrutiny, which would reveal its first-order or second-order nature.

\section{Object language}

We extracted urlogic as a formalization of the act of doing mathematics. The semantics of urlogic is totally informal. However, semantics can be studied 
very well with formal methods. We can define in urlogic a mathematical concept of a language and study it using all the mathematical methods available in urlogic. In this case we call the language thus studied the object language.

Let us assume our urlogic is capable of talking about sets. For simplicity we call it ZFC, although a much weaker urlogic would suffice for the discussion. This urlogic could also be some version of second-order logic, for we argued above that on the level of urlogic there is no essential difference. Some sets code formulas of our intended object language, whatever it happens to be, for example second-order logic or first-order logic. Thus the strings of our urlogic do not only "talk" about sets but also about objects that are finite strings in the sense of the system and denote, again in the sense of the system, first-order or second-order statements. Likewise, some sets code structures. In particular, there are the sets $\mathbb{N}$ and $\mathbb{R}$ that code the structures that result in set theory when one constructs the set of natural numbers and the set of real numbers in some canonical way. Finally, some sets code the truth-definition of the object language, that is, the relation

$\{\langle\mathfrak{A}, \phi\rangle: \mathfrak{A}$ satisfies the sentence $\phi$ of the object language $\}$.

Suppose the object theory is the first-order theory RCF of real closed fields. We can argue in the urlogic ZFC that the structure $\mathbb{R}$ satisfies a given statement $\phi$ of the language of real closed fields. Our argument is totally finitist, but we believe that we prove something about infinite objects like the set of reals, using some finite information about them. Does this mean we could actually dispense with the infinite objects? Does this suggest that infinity is but a blurred vision in our head? This is one of the fundamental questions of the philosophy of mathematics and we have not answered it here one way or another.

Suppose the object theory is the second-order theory $\mathrm{RCF}_{2}$ of real closed fields. We can argue in the urlogic $\mathrm{ZFC}$ that the structure $\mathbb{R}$ is the only model of $\mathrm{RCF}_{2}$. The advantage of $\mathrm{RCF}$ over $\mathrm{RCF}_{2}$ is that it can also be used as a tool for proving the decidability of the first-order theory of the arithmetic of the reals [Tar48].

We can also argue in the urlogic ZFC that the object language satisfies the Gödel Completeness Theorem, i.e. if a sentence $\phi$ of the object language is true in every model of another sentence $\psi$ of the object language, then there is (a set that codes) a finite object language proof of $\phi$ from $\psi$. This 
is the case if the object language is first-order logic, but not the case if it is second-order logic.

Suppose we have an urlogic proof that in the object language there is a proof of $\phi$ from $\psi$ obtained perhaps using the Gödel Completeness Theorem. Can we get out of this a proof of $\phi$ from $\psi$ in the urlogic? In several ways this question is meaningless. However, we may think of ZFC itself as the object language and $\phi$ and $\psi$ as sentences of urlogic. It is then possible to write definitions of sets that code these sentences. Suppose this is done and then we get a proof of $\phi$ from $\psi$ in the object language using the Gödel Completeness Theorem. This still does not give us a proof of $\phi$ from $\psi$ in urlogic, but we have a strong reason to believe that such a proof can be written. This is because the concept of a finite proof is so strongly absolute (see Section 6 below). But it still depends on the concept of finiteness, and a further elaboration, using a third level of formalization, or alternatively an informal model-theoretic argument, shows that a set-theoretical object can be a finite proof in the sense of the object theory but infinite in the sense of urlogic.

On the other hand, suppose we prove in the urlogic that there is a model of $\phi$ in which $\psi$ is false. Now there cannot be a proof of $\psi$ from $\phi$ in the urlogic, since such a proof would be formalizable in the object language. Thus studying models of sentences of a first-order object language can give important information about proofs in the urlogic. If the object language is second-order, this possibility does not exist.

We can try to understand the urlogic by letting the object language be a picture of the urlogic. Some mathematical properties of this object language give us information about the urlogic. To understand which properties reflect between urlogic and object language, we need to formalize the situation and study it using methods of urlogic. So we not only have an object language, but also a formalization of a language in the object language.

Let us consider an example. If the urlogic is ZFC, we can define in ZFC the axiom system ZFC (as a mathematical object) and study its models. In these models of set theory there are also axiomatizations of set theory and their models. The picture is clearer if we consider only transitive models. In a transitive model there can be elements which are transitive models of set theory. We know that it is exactly the ZFC-absolute i.e. $\Delta_{1}^{\mathrm{ZFC}}$-properties of sets which reflect between transitive (in fact, end-extensions of) models of ZFC. If we consider all models, not just the transitive ones, then exactly properties that are existential relative to $\mathbf{Z F C}$ reflect from submodels to 
supermodels. For example, a set may be infinite in $\mathfrak{M}=\mathbf{Z F C}$ but finite in a submodel $\mathfrak{N} \models \mathbf{Z F C}$ of $\mathfrak{M}$. Likewise, a set may be countable in $\mathfrak{M}$ but uncountable in $\mathfrak{N}$. This example shows that we should be careful in reflecting our non-absolute observations about the object theory to urlogic.

It is true that full second-order logic as an object theory has categorical axiomatizations for important mathematical structures. However, these facts are highly non-absolute so we cannot use these facts to motivate the claim that our urlogic has full second-order strength. There are some grounds for such a leap only when we talk about very absolute properties, like "there is a proof of ...". So it is rather the features of the Henkin second-order logic that can be said to be characteristic of the urlogic.

\section{The absoluteness of first-order logic}

An important feature of first-order logic is that it is absolute in a strong sense. Absoluteness has a technical meaning in set theory, which first-order logic fulfills in several ways (see [Bar72] and [V85]). For example, the property $\mathrm{ZFC} \vdash \phi$ is an r.e. property of a first-order sentence $\phi$. Therefore, if ZFC $\vdash \phi$ is true, its formalized version holds inside any model of first-order Peano arithmetic, and the formalized version is provable in Peano arithmetic. On the other hand, if the formalized version of ZFC $\vdash \phi$ is provable in Peano arithmetic, then $\mathrm{ZFC} \vdash \phi$. Thus the absoluteness of first-order logic permits us to reduce questions about $\mathrm{ZFC}$ set theory to questions about first-order number theory.

A consequence of the absoluteness of first-order logic is the LöwenheimSkolem Theorem: If a first-order sentence has for each finite number $n$ a model (perhaps infinite) with at least $n$ elements, then the sentence has models in all infinite cardinalities. It is remarkable that by Lindström's Theorem [Lin69], first-order logic is the only logic with this property. This extreme flexibility of first-order logic with respect to the cardinality of the universe is often held against first-order logic. This flexibility does indeed mean, that first-order logic cannot really say of the reals, for example, that they are uncountably many, without saying it in set theory. Likewise, in what is called the Skolem Paradox, first-order set theory cannot say of its own universe that it is uncountable. But this is, in fact, as it should be. There is nothing wrong in a formal system not being able to talk about its own semantics. Meaning is beyond language. 
The great flexibility of first-order logic has made model theory possible. During the last 50 years model theory has made great advances in classifying first-order theories with a nice structure theory for their models. One of the surprising results of stability theory is that the mere number of nonisomorphic models that a first-order theory has in different cardinalities gives deep information about the geometric and algebraic structure of its models [She82b, She82a].

\section{The expressive power of second-order logic}

Second-order formalization has a lot of appeal for the same reason which makes it, in another sense, unappealing: it is very close to informal reasoning. Mathematicians are used to thinking of natural numbers as a structure categorically defined by the (second-order) Peano axioms, and of reals as the unique complete separable archimedian completely ordered field, and so forth. Mathematical practice is full of categoricity results that can be formalized using second-order logic ([Mon65b]). This is an important feature of the logic. In each case the reasoning remains informal.

Suppose we want to do set theory using second-order logic. Subset existence is built into the language, so we do not need the usual set existence axioms. We still need axioms for iterating the set construction, i.e. forming sets of sets, sets of sets of sets, etc. It is natural to proceed as in first-order set theory with individual variables ranging over sets and second-order variables ranging over subsets of the universe. Let $\mathrm{ZFC}_{2}$ be the resulting second-order version of ZFC (see [Mon65b]). The models of $\mathrm{ZFC}_{2}$ are, up to isomorphism, of the form $R_{\kappa}$, where $\kappa$ is a (strongly) inaccessible cardinal. By adding the appropriate second-order axiom, we can get a theory $\mathrm{ZFC}_{2}^{\mathrm{M}}$, which has as its models exactly sets of the form $R_{\kappa}$, where $\kappa$ is a (strongly) Mahlo cardinal, and $\mathrm{ZFC}_{2}^{\mathrm{WC}}$, which has as its models exactly sets of the form $R_{\kappa}$, where $\kappa$ is a weakly compact cardinal. A well-known consequence of properties of measurable cardinals is that one cannot go on in the same way to $R_{\kappa}$ with $\kappa$ measurable, without at the same time going to third order $\operatorname{logic}^{9}$. Note that $\mathrm{ZFC}_{2}, \mathrm{ZFC}_{2}^{\mathrm{M}}$ and $\mathrm{ZFC}_{2}^{\mathrm{WC}}$ are not equivalent. If there is an inaccessible cardinal, then there is a model of $\mathrm{ZFC}_{2}$, which is not a model of $\mathrm{ZFC}_{2}^{\mathrm{M}}$, and if there is a Mahlo cardinal, then there is a model of $\mathrm{ZFC}_{2}^{\mathrm{M}}$, which is not a model of $\mathrm{ZFC}_{2}^{\mathrm{WC}}$.

\footnotetext{
${ }^{9}$ This has been pointed out by Kreisel [Kre67].
} 
If we add to $\mathrm{ZFC}_{2}$ the axiom "There are no inaccessible cardinals", the resulting system is categorical. Similarly $\mathrm{ZFC}_{2}^{\mathrm{M}}$ and $\mathrm{ZFC}_{2}^{\mathrm{WC}}$ can be strengthened to categorical second-order theories.

What happens to the property $\mathrm{ZFC}_{2}=\phi^{10}$ of a second-order sentence $\phi$, when we move to a formalization of second-order logic? Let us first consider formalization in $\mathrm{ZFC}_{2}$ itself. The formalized version of $\mathrm{ZFC}_{2} \models \phi$ holds in every model of $\mathrm{ZFC}_{2}$ if and only if $\mathrm{ZFC}_{2} \models \phi$. So the property is absolute, but for a (trivial) different reason than in the case of first-order logic. In particular, we achieved no reduction at all. In order to achieve some reduction, let us try to formalize $\mathrm{ZFC}_{2} \models \phi$ in second-order number theory. How are we to talk about structures $R_{\kappa}$, where $\kappa$ is inaccessible, using just natural numbers and sets of natural numbers? This does not seem possible. Let us then consider the predicate $P_{2} \models \phi$, where $P_{2}$ is the full second-order number theory ([Mon65b]) and $\phi$ is a sentence of second-order number theory. As $P_{2}$ has, up to isomorphism, only one model, $P_{2}=\phi$ really means $\mathbb{N} \models \phi$. In $\mathbb{N}$ there are just natural numbers, so we cannot talk ${ }^{11}$ about models of $P_{2}$. What would work, is talking about $P_{2}=\phi$ in the full second-order arithmetic $A_{2}$. Then we get absoluteness. The property $P_{2} \models \phi$ is absolute relative to the model(s) of $A_{2}$. But this is uninteresting as $A_{2}$ is stronger than $P_{2}$. In the case of first-order logic we got absoluteness (of ZFC $\vdash \phi$ ) relative to a weaker theory (viz. number theory), which could genuinely be called a reduction.

A feature of second-order set theories is that they are categorical. What do these theories tell us? They tell us, that if we know what the universe is like, we can pick the corresponding second-order categorical axiomatization of it. The problem is of course, that we do not know and will never know. The most informative use of second-order axiomatizations seems to be in the framework of first-order set theory. The categorical second-order theories pick certain interesting invariant structures among the many standard models of set theory.

What do the various second-order set theories (i.e. models) tell us about the Continuum Hypothesis $(\mathrm{CH})$ ? They tell us that if $\mathrm{CH}$ is true, then it is true in these models. If $\mathrm{CH}$ is false, then it is false in these models. The difference between this and the first-order ZFC is that we can prove, using low level formalization, that whether $\mathrm{CH}$ is true or not, $\mathrm{ZFC}$ cannot prove

\footnotetext{
${ }^{10}$ I.e. $\phi$ is true in every model of $\mathrm{ZFC}_{2}$

${ }^{11}$ In a trivial sense then the formalized version of $P_{2}=\phi$ could be considered true by default. But this argument does not work in the other direction, if the formalized version of $P_{2} \models \phi$ is true (by default) in $\mathbb{N}$, it does not follow that $P_{2} \models \phi$.
} 
or disprove it. This is a clear message that new axioms are needed for ZFC. In contrast, the categorical second-order theories do not seem to give us any clue as to what to do next.

Let $V a l^{2}$ be the set of Gödel numbers $\ulcorner\phi\urcorner$ of valid second-order sentences $\phi$ in a vocabulary that contains one binary predicate symbol $P$. It is known that $V a l^{2}$ is a highly complex subset of $\mathbb{N}$. For example, $V a l^{2}$ is not $\Sigma_{n}^{m}$ for any $m, n<\omega$ ([Mon65a], [Hin55]). What exactly is the complexity of this set?

Theorem $1 \mathrm{Val}^{2}$ is the complete $\Pi_{2}$-definable ${ }^{12}$ set of integers.

Proof. Let us first observe that the predicate $x=\mathcal{P}(y)$ is $\Pi_{1}$-definable. We can also $\Pi_{1}$-define the property $R(x)$ of $x$ of being equal to some $R_{\kappa}$, where $\kappa$ is a strong limit cardinal. Let $\operatorname{Str}(x)$ be the first-order formula in the language of set theory which says that $x$ is a structure of the vocabulary containing just one binary predicate symbol. If $\phi$ is a second-order sentence, let $\operatorname{Sat}_{\phi}(x)$ be the first-order formula in the language of set theory which says "Str $(x)$ and $\phi$ is true in the structure $x$ ", and let $\operatorname{Relsat}_{\phi}(x, y)$ be the first-order formula in the language of set theory which says " $x \in y$ and if $\operatorname{Str}(x)$, then $\operatorname{Sat}_{\phi}(x)$ is true when relativized to the set $y$ ". Thus $\ulcorner\phi\urcorner \in V a l^{2}$ if and only if $\forall x\left(\operatorname{Str}(s) \rightarrow \operatorname{Sat}_{\phi}(x)\right)$. Note that for limit $\alpha$ and $a \in R_{\alpha}$ :

$$
\operatorname{Sat}_{\phi}(a) \Longleftrightarrow\left(R_{\alpha} \models \operatorname{Sat}_{\phi}(a)\right) .
$$

Thus a second-order sentence $\phi$ is valid if and only if

$$
\forall x\left(R(x) \rightarrow \forall y \in x \operatorname{Relsat}_{\phi}(y, x)\right) .
$$

We have proved that $\mathrm{Val}^{2}$ is $\Pi_{2}$-definable. Suppose then $A$ is an arbitrary $\Pi_{2}$-definable set of integers. Let $\forall x \exists y \psi(n, x, y)$ be the $\Pi_{2}$-definition. Let $\phi_{n}$ be the first-order sentence $\forall x \exists y \psi(n, x, y)$, where $n$ is a defined term. We claim

$$
n \in A \Longleftrightarrow\left\ulcorner\mathrm{ZFC}_{2}^{-} \rightarrow \phi_{n}\right\urcorner \in V a l^{2},
$$

where $\mathrm{ZFC}_{2}^{-}$is a finite second-order axiomatization of the models $R_{\kappa}, \kappa$ strong limit. Suppose first $n \in A$, i.e. $\forall x \exists y \psi(n, x, y)$. Suppose $R_{\kappa}$ is a given model of $\mathrm{ZFC}_{2}^{-}$. We prove $R_{\kappa} \models \forall x \exists y \psi(n, x, y)$. Suppose $a \in R_{\kappa}$. By

\footnotetext{
${ }^{12}$ We mean definability in set theory by a formula of the form $\forall y \exists z \phi(x, y, z)$, where $\phi(x, y, z)$ is quantifier-free.
} 
the Levy Reflection Principle, there is $b \in R_{\kappa}$ such that $\psi(n, a, b)$. Hence $\left\ulcorner\mathrm{ZFC}_{2}^{-} \rightarrow \phi_{n}\right\urcorner \in V a l^{2}$. Conversely, suppose $\left\ulcorner\mathrm{ZFC}_{2}^{-} \rightarrow \phi_{n}\right\urcorner \in V a l^{2}$. To prove $\forall x \exists y \psi(n, x, y)$, let $a$ be given. Let $\kappa$ be a strong limit cardinal such that $a \in R_{\kappa}$. Then there is $b \in R_{\kappa}$ with $R_{\kappa} \models \psi(n, a, b)$. Now $\psi(n, a, b)$ follows.

Corollary $2 \mathrm{Val}^{2}$ is not $\Sigma_{2}$-definable.

The fact that $V a l^{2}$ is not $\Sigma_{n}^{m}$ for any $m, n<\omega$ follows easily from this. Moreover, it follows that we cannot in general express " $\phi$ is valid", for secondorder $\phi$, even by searching through the whole set-theoretical universe for a set $x$ such that a universal quantification over the subsets of $x$ would guarantee the validity of $\phi$. In contrast, to check validity of a first-order sentence, one needs only search through all natural numbers and then perform a finite polynomial calculation on that number.

\section{IF-logic}

Hintikka has suggested that the so-called IF-logic provides a new foundation for mathematics [Hin96]. IF-logic is an extension of first-order logic which is semantically equivalent with the $\Sigma_{1}^{1}$-part of second-order logic. The secondorder theories $P_{2}, A_{2}$ and $\mathrm{ZFC}_{2}$ can all be (finitely) axiomatized in the $\Pi_{1}^{1}$ part of second-order logic. Thus, if we want to know whether, say, $\mathrm{ZFC}_{2}=\phi$, where $\phi$ is first-order, all we have to do is to check whether $\left(\neg \bigwedge \mathrm{ZFC}_{2}\right) \vee \phi$ is valid in IF-logic. Now IF-logic has the nice property, by virtue of its relation to the $\Sigma_{1}^{1}$-part of second-order logic, that the set of Gödel numbers of satisfiable sentences is $\Pi_{1}^{0}$. So this is the same as with first-order logic. We have a logic with a $\Pi_{1}^{0}$-concept of satisfiability and such important questions as whether $\mathrm{ZFC}_{2} \models \phi$, can be reduced to validity in the logic. This seems like an interesting reduction of set theory, and thereby all of mathematics, to validity in pure IF logic.

Hintikka argues that validity in IF-logic is a combinatorial question, rather than a set theoretic question; it is the question, whether a certain relational structure cannot help being instantiated in every model [Hin96, p. 198]. In the case of $P_{2} \models \phi$, since $P_{2}$ has but one model, it is the question whether $\phi$ is true in $\mathbb{N}$ or not. For $\mathrm{ZFC}_{2} \models \phi$, it is the question whether $\phi$ is true in every $R_{\kappa}, \kappa$ inaccessible, or not. 
The following question arises: Suppose we formalize the inference $P_{2} \models \phi$ into something, let us call it $\left\ulcorner P_{2} \models \phi\right\urcorner$. The goal of formalization is to be able to say something of the nature of the inference. We are not satisfied with the information: $\left\ulcorner P_{2} \models \phi\right\urcorner$ if and only if $P_{2} \models \phi$, but want something more. The question is, what more does formalization $\left\ulcorner P_{2} \models \phi\right\urcorner$ in IF-logic tell us than just $P_{2}=\phi$ ? In comparison, first-order logic gives the analysis: $\ulcorner P \models \phi\urcorner$ if and only if there is a finite proof of $\phi$ from $P$.

It follows from the preceding discussion that if $\operatorname{Val}_{I F}$ denotes the set of Gödel numbers of valid sentences of IF-logic with at least one binary predicate symbol, then:

Theorem $3 \mathrm{Val}_{I F}$ is recursively isomorphic with $\mathrm{Val}^{2}$.

Corollary $4 \mathrm{Val}_{I F}$ is the complete $\Pi_{2}$-definable set of integers, hence Val ${ }_{I F}$ is not $\Sigma_{n}^{m}$ for any $m, n<\omega$.

This shows that also in a technical sense IF-logic is as complicated as the full second-order logic.

It is difficult to see how IF-logic would work as a foundation for mathematics, apart from the way second-order logic itself works. We have argued above that second-order logic as urlogic is indistinguishable from first-order logic and the same seems true of IF-logic.

\section{Conclusion}

Whatever is proved from $\mathrm{ZFC}$ can be turned into an informal argument in second-order logic, e.g. in $\mathrm{ZFC}_{2}$. On the other hand, if something can be informally argued in $\mathrm{ZFC}_{2}$, it seems very likely, that behind the informal argument is an argument that can be formalized in ZFC. In this respect there is very little difference between first-order set theory and second-order logic. The same applies to IF-logic.

On the other hand, if we try to analyse why we are not able to decide e,g, Continuum Hypothesis, on the basis of $\mathrm{ZFC}_{2}$, it seems very plausible to develop a theory about what the second-order quantifiers range over. The first-order set theory ZFC is exactly such a theory, and it is indeed the strongest currently available tool for investigating formalizations of secondorder logic. But this means we are back in the Henkin semantics of secondorder logic that full second-order logic was supposed to avoid. 


\section{References}

[Bar72] K. Jon Barwise. Absolute logics and $L_{\infty \omega}$. Ann. Math. Logic, 4:309-340, 1972.

[Hen50] Leon Henkin. Completeness in the theory of types. J. Symbolic Logic, 15:81-91, 1950.

[Hin55] Jaakko Hintikka. Reductions in the theory of types. Acta Philos. Fenn., 8:57-115, 1955.

[Hin96] Jaakko Hintikka. The principles of mathematics revisited. Cambridge University Press, Cambridge, 1996. With an appendix by Gabriel Sandu.

[Kre67] G. Kreisel. Informal rigour and completeness proofs. In Proceedings of the International Colloquium in the Philosophy of Science, London, 1965, Vol. 1. Edited by Imre Lakatos, pages 138-157. North-Holland Publishing Co., Amsterdam, 1967.

[Lin69] Per Lindström. On extensions of elementary logic. Theoria, 35:111, 1969.

[Lin73] Per Lindström. A note on weak second order logic with variables for elementarily definable relations. In The Proceedings of the Bertrand Russell Memorial Conference (Uldum, 1971), pages 221-233. Bertrand Russell Memorial Logic Conf., Leeds, 1973.

[Mon65a] Richard Montague. Reduction of higher-order logic. In Theory of Models (Proc. 1963 Internat. Sympos. Berkeley), pages 251-264. North-Holland, Amsterdam, 1965.

[Mon65b] Richard Montague. Set theory and higher-order logic. In Formal Systems and Recursive Functions (Proc. Eighth Logic Colloq., Oxford, 1963), pages 131-148. North-Holland, Amsterdam, 1965.

[Mos61] Andrzej Mostowski. Concerning the problem of axiomatizability of the field of real numbers in the weak second order logic. In Essays on the foundations of mathematics, pages 269-286. Magnes Press, Hebrew Univ., Jerusalem, 1961. 
[Sha91] Stewart Shapiro. Foundations without foundationalism. The Clarendon Press Oxford University Press, New York, 1991. A case for second-order logic, Oxford Science Publications.

[She82a] Saharon Shelah. The spectrum problem. I. $\aleph_{\varepsilon}$-saturated models, the main gap. Israel J. Math., 43(4):324-356, 1982.

[She82b] Saharon Shelah. The spectrum problem. II. Totally transcendental and infinite depth. Israel J. Math., 43(4):357-364, 1982.

[Sim99] Stephen G. Simpson. Subsystems of second order arithmetic. Springer-Verlag, Berlin, 1999.

[Tar48] Alfred Tarski. A Decision Method for Elementary Algebra and Geometry. RAND Corporation, Santa Monica, Calif., 1948.

[Vää85] Jouko Väänänen. Set-theoretic definability of logics. In Modeltheoretic logics, pages 599-643. Springer, New York, 1985. 